\title{
Protective effects of tumor necrosis factor- $\alpha$ blockade by adalimumab on articular cartilage and subchondral bone in a rat model of osteoarthritis
}

\author{
C.H. $\mathrm{Ma}^{1 *}$, Q. $\mathrm{Lv}^{2 *}, \mathrm{Y} . \mathrm{X} . \mathrm{Yu}^{1}, \mathrm{Y} . \mathrm{Zhang}^{1}$, D. Kong ${ }^{1}$, K.R. Niu ${ }^{1}$ and C.Q. Yi ${ }^{1}$ \\ ${ }^{1}$ Department of Orthopedic Surgery, Shanghai First People's Hospital, Shanghai Jiao Tong University, Shanghai, China \\ ${ }^{2}$ Department of Radiology, Tong Ji Hospital, Tong Ji University, Shanghai, China
}

\begin{abstract}
We aimed to investigate the effects of an anti-tumor necrosis factor- $\alpha$ antibody (ATNF) on cartilage and subchondral bone in a rat model of osteoarthritis. Twenty-four rats were randomly divided into three groups: sham-operated group $(n=8)$; anterior cruciate ligament transection (ACLT) + normal saline (NS) group $(n=8)$; and ACLT+ATNF group ( $n=8)$. The rats in the ACLT + ATNF group received subcutaneous injections of ATNF $(20 \mu \mathrm{g} / \mathrm{kg})$ for 12 weeks, while those in the ACLT + NS group received NS at the same dose for 12 weeks. All rats were euthanized at 12 weeks after surgery and specimens from the affected knees were harvested. Hematoxylin and eosin staining, Masson's trichrome staining, and Mankin score assessment were carried out to evaluate the cartilage status and cartilage matrix degradation. Matrix metalloproteinase (MMP)-13 immunohistochemistry was performed to assess the cartilage molecular metabolism. Bone histomorphometry was used to observe the subchondral trabecular microstructure. Compared with the rats in the ACLT + NS group, histological and Mankin score analyses showed that ATNF treatment reduced the severity of the cartilage lesions and led to a lower Mankin score. Immunohistochemical and histomorphometric analyses revealed that ATNF treatment reduced the ACLT-induced destruction of the subchondral trabecular microstructure, and decreased MMP-13 expression. ATNF treatment may delay degradation of the extracellular matrix via a decrease in MMP-13 expression. ATNF treatment probably protects articular cartilage by improving the structure of the subchondral bone and reducing the degradation of the cartilage matrix.
\end{abstract}

Key words: Anti-tumor necrosis factor- $\alpha$ antibody (ATNF); Osteoarthrosis; Matrix metalloproteinase (MMP)-13; Articular cartilage; Subchondral bone

\section{Introduction}

Osteoarthritis $(\mathrm{OA})$ is a highly prevalent aging-associated degenerative joint disorder in middle-aged and older people around the world (1). Clinically, the disease is characterized by progressive degeneration of articular cartilage with inflammation in the synovium, subchondral bone sclerosis, and marginal osteophyte formation (2). Although many factors contribute to the onset of OA, including genetic, metabolic, biochemical, and biomechanical factors $(3,4)$, the exact pathogenesis of OA remains unknown (5). The main approach in current OA therapies is drug treatment to relieve pain and improve joint function. However, there are no therapeutic strategies that can address the underlying causes to halt OA progression.

Chondrocytes and extracellular matrix (ECM) are the pivotal structural components of cartilage (6). OA is considered to start as a result of damage to the joint tissue by physical forces such as single or repeated microtrauma (7). Chondrocytes respond to such physical injury by stopping the production of anabolic factors and by releasing more catabolic enzymes such as matrix metalloproteinases (MMPs). These responses result in further damage to the cartilage (8), and consequently lead to the release of matrix components, which elicit inflammatory mechanisms. Moreover, breakdown of the ECM eventually presents as articular cartilage degeneration (6). Studies have revealed that the pathophysiology of OA involves proinflammatory cytokines, such as interleukin$1 \beta$, tumor necrosis factor (TNF)- $\alpha$, and interleukin-6 (9). The role of $T N F-\alpha$ in the pathogenesis of OA has drawn increasing attention in recent years because of its

Correspondence: Chengqing Yi: <dryichengqing@163.com>.

${ }^{*}$ C.H. Ma and Q. Lv are co-first authors.

Received November 10, 2014. Accepted March 2, 2015. First published online July 31, 2015. 
predominance in the pathogenesis of other arthritic diseases (10-12). By retarding joint damage, approaches that target TNF- $\alpha$ blockade may provide effective therapies for OA.

Adalimumab (D2E7; Abbott Laboratories, USA) is the first fully human (100\% human peptide sequences) monoclonal antibody that blocks TNF- $\alpha$. Regarding rheumatoid arthritis (RA), adalimumab is currently being evaluated in clinical trials because it can slow progressive joint destruction $(13,14)$. Few studies have explored the effects of adalimumab therapy on OA in clinical settings. Verbruggen et al. (15) showed that adalimumab significantly delayed the progression of joint damage in patients with erosive hand OA. However, the mechanism underlying the effects of adalimumab on OA is largely unknown. Therefore, further studies are needed to clarify the exact effects of adalimumab on OA.

The anterior cruciate ligament transection (ACLT) model is one of the most widely used models of OA, and mimics early OA in humans very well (16-18). In the present study, we examined the potential effects of an anti-TNF- $\alpha$ antibody (ATNF) by observing the changes in the chondrocytes, ECM, and subchondral trabecular bone in the rat ACLT model of OA. We aimed to explore the potential mechanism underlying the effects of ATNF on OA. We hypothesized that ATNF could affect TNF- $\alpha-$ induced MMP-13 expression and improve the subchondral bone microstructure to inhibit cartilage degeneration and alter the subchondral bone quality. Our study can provide a theoretical basis for the potential effects of ATNF on $O A$ in clinical trials.

\section{Material and Methods}

\section{Animal handling and ACLT surgery}

All procedures were approved by the Animal Care and Ethics Committee of Shanghai First People's Hospital, Shanghai Jiao Tong University. A total of 24 healthy female Sprague-Dawley rats (aged 11 weeks, weighing $272.5 \pm 38.5 \mathrm{~g}$; Charles River Corporation, China; Batch No. SCXK Beijing, 2007-0001) were used in the following experiments. The rats were randomly separated into three groups of eight rats as follows: sham-operated (SP), ACLT + normal saline (NS) (ACLT + NS); and ACLT + ATNF.

The rats in the ACLT+ATNF and ACLT + NS groups underwent ACLT surgery, while the rats in the SP group underwent knee joint exposure only, followed by suturing. The ACLT model was created as previously described (19). Briefly, each rat in the ACLT + ATNF and ACLT + NS groups was anesthetized intraperitoneally with $10 \%$ chloral hydrate (30 mg/kg) prior to surgery. Their right legs were prepared and draped in a standard sterile manner, before an approximately 2-cm midline incision was made over the knee. The patella was dislocated laterally and the knee placed in full flexion. The anterior cruciate ligament was transected with micro-scissors, and complete transection was confirmed by the anterior drawer test. For surgery in the SP group, the knee joint space was exposed, but the anterior cruciate ligament was not transected. Following surgery, the capsule and skin were both sutured, and the skin was disinfected with iodine.

At 3 days after surgery, the rats were given an antibiotic $(50,000 \mathrm{U}$ penicillin/rat) every day with appropriate postoperative care and allowed to exercise freely. After 1 week, the rats in the ACLT + ATNF group were given ATNF (adalimumab) treatment every 2 days by subcutaneous injection at a dosage of $20 \mu \mathrm{g} / \mathrm{kg}$. The rats in the ACLT + NS group were injected with NS using the same volume as the ACLT + ATNF group. The rats were checked daily (activity, body weight, food consumption, rectal temperature, wound healing). All animals were treated for 12 weeks and then euthanized.

\section{Specimen collection}

The rats were euthanized by cervical dislocation and their right knee joint spaces were opened. The gross appearance of the distal femur was observed and recorded with a digital camera (Model 550D; Canon, Japan). Specimens of the femurs were then fixed in $4 \%$ paraformaldehyde for $72 \mathrm{~h}$, treated with $15 \%$ disodium ethylenediaminetetraacetic acid, and embedded in paraffin. The embedded tissues were sectioned at 4- $\mu \mathrm{m}$ thickness and processed for conventional staining, specific staining, and immunohistochemistry.

In addition, specimens of the proximal end of the tibias were fixed in $70 \%$ ethanol for $72 \mathrm{~h}$. The tissues were then dehydrated, embedded in methyl methacrylate, cut into $5-\mu \mathrm{m}$ thick sections, and subjected to von Kossa staining. Using light microscopy, two color digital images were recorded to analyze the articular cartilage lesions in sections from different regions. For analysis of morphological changes to the cartilage, the Mankin score system was properly adjusted and applied.

\section{Bone histomorphometry}

Undecalcified samples were subjected to von Kossa staining (20). Histomorphometric measurements were determined with a DMLB2 fluorescence/light microscope (Leica, Germany) and a DC300 figure shoot system (Leica, Germany). A Leica Qwin image analysis system with automated analysis was used for evaluation of standard morphological parameters, including bone volume fraction (BV/TV), trabecular bone thickness (Tb.Th), trabecular bone number (Tb.N), and trabecular bone separation (Tb.Sp).

\section{Hematoxylin and eosin staining}

Sections were deparaffinized by sequential immersion in xylene, rehydrated in solutions of absolute alcohol $(95 \%, 80 \%$, and $70 \%$ alcohol), and briefly washed in distilled water. The sections were stained in Harris hematoxylin solution for $5 \mathrm{~min}$, differentiated in $0.5 \%$ acid 
alcohol for $1 \mathrm{~min}$, and stained blue in ammonia water. Counterstaining was performed in eosin solution for $1 \mathrm{~min}$. The stained sections were dehydrated through a graded series of alcohol, cleared with xylene, and mounted with neutral balsam. The morphology of the tissues was observed using an Olympus BX40 light microscope (Olympus, Japan). The sections were scored using slightly modified Mankin criteria (21) (Table 1).

\section{Masson's trichrome staining}

Masson's trichrome staining was performed as described previously (22). Briefly, the sections were deparaffinized, hydrated to distilled water, oxidized in $1 \%$ potassium permanganate solution, and rinsed with tap water. After bleaching with oxalic acid for $1 \mathrm{~min}$, washing in distilled water, and staining in Celestine blue for $5 \mathrm{~min}$, the sections were stained in Mayer's hematoxylin solution for 3-5 min and rinsed in running tap water for 5-10 min. The sections were then stained in Ponceau-picric acid saturated solution for $5 \mathrm{~min}$, rinsed in 1\% acetic acidwater, differentiated in $1 \%$ phosphomolybdic acid for $5 \mathrm{~min}$, rinsed in distilled water, stained in 1\% light green or toluidine blue for $30 \mathrm{~s}$, rinsed in $1 \%$ acetic acid-water, differentiated in 95\% alcohol, hydrated in absolute alcohol, cleared with xylene, and mounted with neutral balsam.

\section{Immunohistochemical staining of MMP-13}

To clarify the molecular mechanism underlying cartilage degeneration, MMP-13 expression was detected by

Table 1. Modified Mankin histological scores.

\begin{tabular}{lc}
\hline Category & Score \\
\hline Structure & \\
Normal & 0 \\
Surface irregularities & 1 \\
Pannus and surface irregularities & 2 \\
Clefts to transitional zone & 3 \\
Clefts to calcified zone & 4 \\
Complete disorganization & 5 \\
Cells & \\
Normal & 0 \\
Diffuse hypercellularity & 1 \\
Clusters & 2 \\
Hypocellularity & 3 \\
Matrix staining & \\
Normal & 0 \\
Slight reduction & 1 \\
Moderate reduction & 2 \\
Severe reduction & 3 \\
No staining & 4 \\
Tidemark integrity & \\
Intact & 0 \\
Destroyed & 1 \\
\hline
\end{tabular}

immunohistochemical staining. Paraffin sections were baked for $20 \mathrm{~min}$, routinely deparaffinized, hydrated to distilled water, and washed three times in phosphatebuffered saline (PBS) for 3 min each. The sections were then treated with complex enzyme digestion for antigen retrieval for $15 \mathrm{~min}$ at room temperature, incubated with $3 \% \mathrm{H}_{2} \mathrm{O}_{2}$ solution for $10 \mathrm{~min}$, and washed three times with PBS for 3 min each. Next, the sections were overlaid with $30 \mu \mathrm{L}$ of anti-mouse MMP-13 polyclonal antibody (Boster Corporation, China) for $10 \mathrm{~min}$ at room temperature, washed three times with PBS for 3 min each, incubated with $30 \mu \mathrm{L}$ of biotinylated goat anti-mouse secondary antibody (DAKO, Denmark) for $40 \mathrm{~min}$, washed three times with PBS for 3 min each, stained with $50 \mu \mathrm{L}$ of 3,3'-diaminobenzidine solution with appropriate termination, and rinsed in tap water for $3 \mathrm{~h}$. Counterstaining was carried out with hematoxylin for $5 \mathrm{~min}$. The sections were then dehydrated through a graded series of alcohol for $10 \mathrm{~s}$ each, cleared with xylene, and mounted with neutral balsam. Images magnified 400-fold were obtained, and the integrated absorbance was determined by Image Pro Plus (IPP) software (Media Cybernetics, USA) to quantify the protein expression.

\section{Statistical analysis}

Results were analyzed using the Shapiro-Wilk normality test (23) and Bartlett homogeneous variance test (24). Student's $t$-test was used to assess the statistical differences among groups and Duncan's multiple-range test was used for values of two individuals. Data were reported as means $\pm S D$. Values of $P<0.05$ were considered to indicate statistical significance.

\section{Results}

\section{Gross macroscopic assessment}

All animals recovered quickly after surgery, and there were no obvious differences in body weight of the rats among the SP, ACLT + ATNF, and ACLT + NS groups (data not shown). The gross appearances were recorded, as shown in Figure 1.

In the SP group, the knees of the rats exhibited translucent smooth articular surface, resembling healthy cartilage, and no osteophyte formation in the femoral condyles or tibial plateaus. The synovial fluid of the knee joints was clear (Figure 1A). In the ACLT + NS group, the knee joints swelled, the cartilage displayed rougher surface, local ulceration and erosion, the synovial fluid was yellow and opaque, the femoral condyles appeared to be hypertrophic, and osteophytes could be found, comprising the typical appearance of OA (Figure 1B). In the ACLT+ATNF group, the majority of the cartilage surface showed slightly rough appearance without osteophyte formation, the synovial fluid was light yellow, and cartilage degradation was decreased compared with the ACLT + NS group (Figure 1C). Overall, the joints in the 

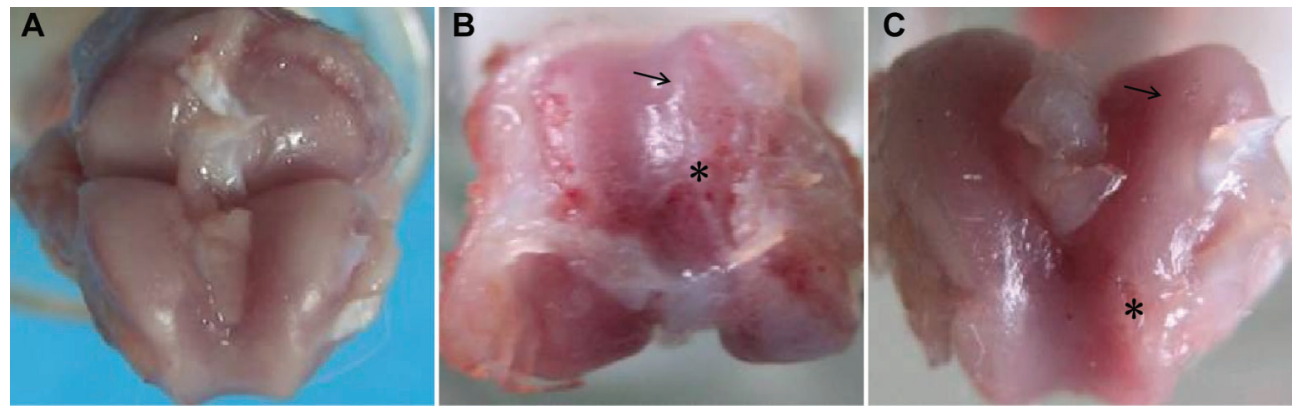

Figure 1. Representative images of femoral condyles at 12 weeks after surgery. $A$, The sham-operated (SP) group shows a normal appearance. $B$, In the anterior cruciate ligament transection (ACLT) + normal saline (NS) group with NS treatment after ACLT, the joints developed surface damage (arrow) and severe osteophytes (asterisk). C, The ACLT + anti-tumor necrosis factor- $\alpha$ antibody (ATNF) group shows a significant reduction in surface damage (arrow) and smaller osteophytes (asterisk).

ACLT + ATNF group showed moderate cartilage degeneration compared to the ACLT + NS group.

\section{Histomorphometric analysis of subchondral bone}

Von Kossa staining was performed for bone histomorphometric analysis. Compared with the ACLT + NS group, $\mathrm{BV} / \mathrm{TV}$ and Tb.N of the rats in the ACLT + ATNF group were significantly increased while Tb.Sp was markedly decreased $(P<0.05)$. However, no significant differences in these parameters were observed between the SP group and the ACLT + ATNF group (Table 2).

\section{Cartilage cell morphology}

Hematoxylin and eosin (HE)-stained sections were used to score samples for features of cartilage pathology, including changes in cellularity and structural abnormalities (Figure 2). In the SP group, the cartilage of the rats appeared as a thick neatly arranged layer, and the staining was normal. The boundary between the calcified cartilage and subchondral bone was intact. In the ACLT+NS group, cartilage lesions were severe. The cartilage layer was thinner, with cell loss, cell cloning, and multicellular chondrocyte clusters, and overall the cells appeared in a less ordered structure. The subchondral bone invaded the calcified cartilage. However, in the ACLT + ATNF group, there were no significant lesions, slight erosion was observed in the cartilage surface, and the cells re-established an ordered pattern, in which the cells were increased in the superficial zone and flattened in the transitional zone. Taken together, these changes resulted in Mankin grades reflected by the analyses shown in Figure 3A.

The Mankin score in the ACLT + NS group was dramatically higher than that in the SP group, and significantly higher than that in the ACLT + ATNF group.

\section{Cartilage matrix morphology}

The cartilage ECM alterations were evaluated by Masson's trichrome staining (Figure 2). Masson trichrome commonly stains the cartilage matrix blue, the nuclei dark blue, and the zone of calcifying cartilage red. We found that the SP group had a regular cell arrangement and dark staining. In the ACLT + NS group, red staining was found, the matrix was strongly but unevenly stained, and the cells had an irregular arrangement. In the ACLT + ATNF group, the cartilage matrix was slightly and unevenly stained, the cells were in an ordered arrangement, and red staining was reduced in the articular cartilage compared with that in the ACLT + NS group.

\section{Immunohistochemical analysis}

Immunohistochemical staining for MMP-13 expression is shown in Figure 4. Staining for MMP-13 was less detectable in the SP group. In the ACLT + ATNF group,

Table 2. Bone histomorphometric analysis of subchondral trabecular bone.

\begin{tabular}{lrrr}
\hline Group & \multicolumn{1}{c}{ SP } & \multicolumn{1}{c}{ ACLT + NS } & \multicolumn{1}{c}{ ACLT+ATNF } \\
\hline BV/TV $(\%)$ & $42.60 \pm 2.60$ & $34.45 \pm 2.73^{*}$ & $44.95 \pm 3.68^{+}$ \\
Tb.N $(\# / \mathrm{mm})$ & $2.90 \pm 1.73$ & $2.48 \pm 0.16^{*}$ & $3.09 \pm 0.30^{+}$ \\
Tb.Th $(\mu \mathrm{m})$ & $146.64 \pm 2.17$ & $138.29 \pm 2.52^{*}$ & $145.36 \pm 7.02^{+}$ \\
Tb.Sp $(\mu \mathrm{m})$ & $198.32 \pm 20.00$ & $264.73 \pm 29.96^{*}$ & $179.72 \pm 27.64^{+}$ \\
\hline
\end{tabular}

BV/TV: bone volume fraction; Tb.N: trabecular number; Tb.Th: trabecular thickness; Tb.Sp: trabecular separation; ATNF: anti-tumor necrosis factor- $\alpha$ antibody. ${ }^{*} \mathrm{P}<0.05$, compared with the sham-operated (SP) group; ${ }^{+} \mathrm{P}<0.05$, compared with the anterior cruciate ligament transection (ACLT) + normal saline (NS) group (Student's $t$-test). 

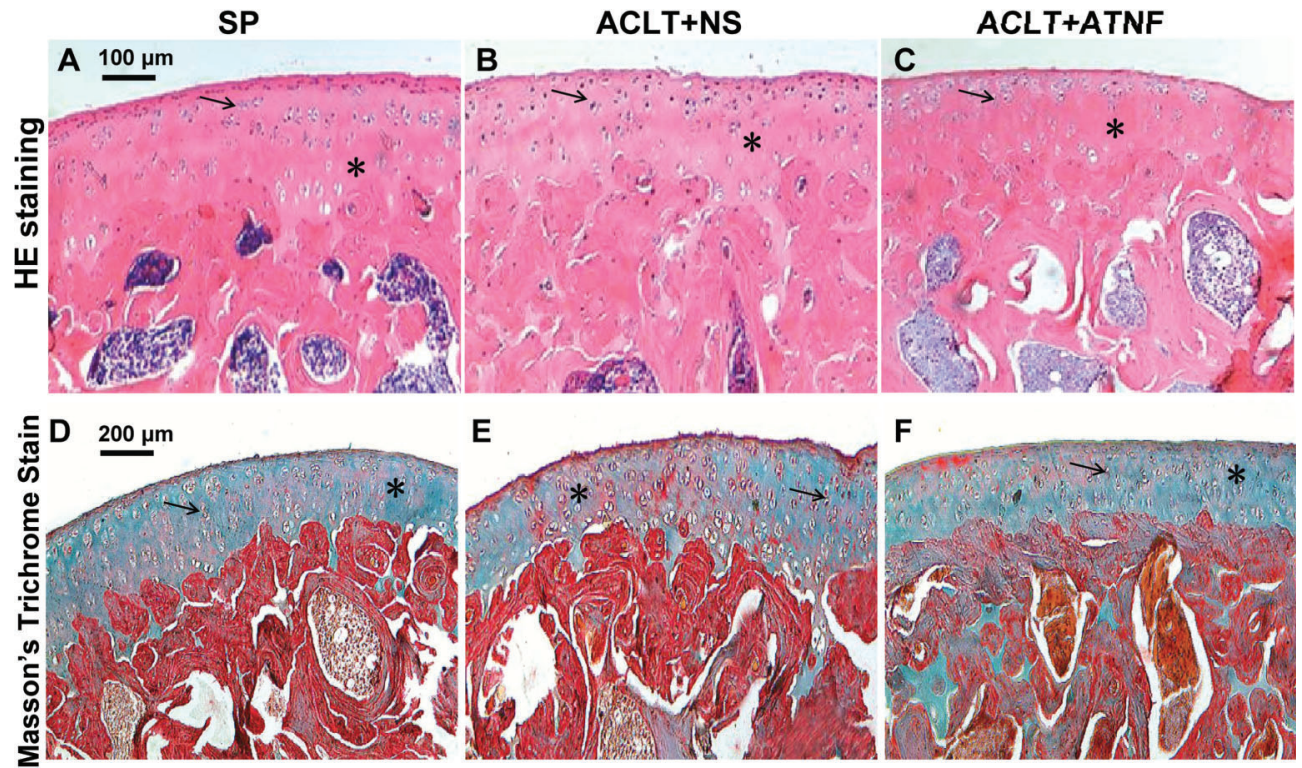

Figure 2. Histological comparison of articular cartilage findings at the distal femur by hematoxylin and eosin (HE) staining (magnification 200x) and cartilage matrix morphology findings by Masson's trichrome staining (magnification 100x). $A, D$, sham-operated (SP) group; $B, E$, anterior cruciate ligament transection (ACLT) + normal saline (NS) group; $C, F, A C L T+$ anti-tumor necrosis factor- $\alpha$ antibody (ATNF) group. In the ACLT + NS group, the cells had an irregular arrangement. In the ACLT + ATNF group, the cells re-established an ordered pattern and the cartilage matrix was slightly and unevenly stained. Arrows indicate the cells. Asterisks represent the cartilage matrix.

MMP-13 was mainly detected in chondrocytes at and close to the articular surfaces (Figure $4 C$ ). In the $\mathrm{ACLT}+\mathrm{NS}$ group as a control, MMP-13 expression was found throughout the articular cartilage. In the ACLT+ ATNF group, ATNF treatment reduced the expression of MMP-13 in cartilage and the integrated absorbance values of the positive cells in the cartilage of rats in the SP group were markedly lower than those in the ACLT+NS group. The integrated absorbance values of positive cells in the cartilage of the ACLT + ATNF group were reduced compared with those in the ACLT + NS group (Figure 3B).

\section{Discussion}

$\mathrm{OA}$ is a common joint disease in the elderly and impedes their daily life. Degenerative alterations to the cartilage and subchondral bone play key roles in OA development (25). Our study demonstrated that ATNF treatment can inhibit cartilage degradation by decreasing MMP-13 expression related to the modulation of cartilage metabolism in a rat model of OA. In addition, ATNF treatment ameliorated the subchondral trabecular bone alterations in the knee joints induced by
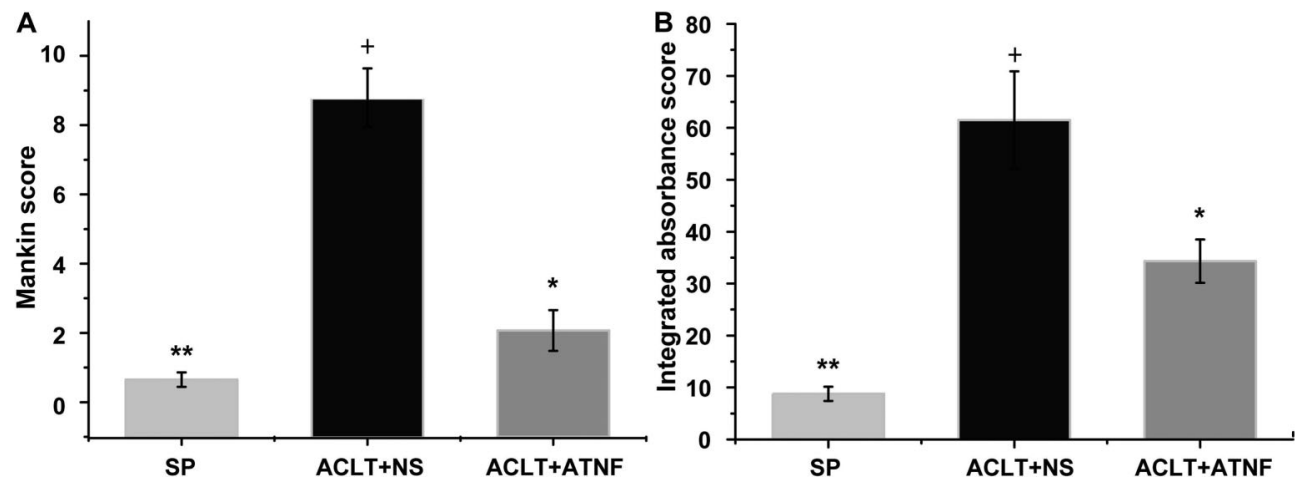

Figure 3. $A$, Mankin scores for grading of cartilage lesions. $B$, integrated absorbance values reflecting matrix metalloproteinase (MMP)13 expression. ${ }^{+} \mathrm{P}<0.05$, anterior cruciate ligament transection (ACLT) + normal saline (NS) group compared to the sham-operated (SP) group; * $\mathrm{P}<0.05, \mathrm{ACLT}+$ anti-tumor necrosis factor- $\alpha$ antibody (ATNF) group compared to the $\mathrm{ACLT}+\mathrm{NS}$ group; ${ }^{* *} \mathrm{P}<0.05$, SP group compared to the ACLT + ATNF group (Student's $t$-test). 


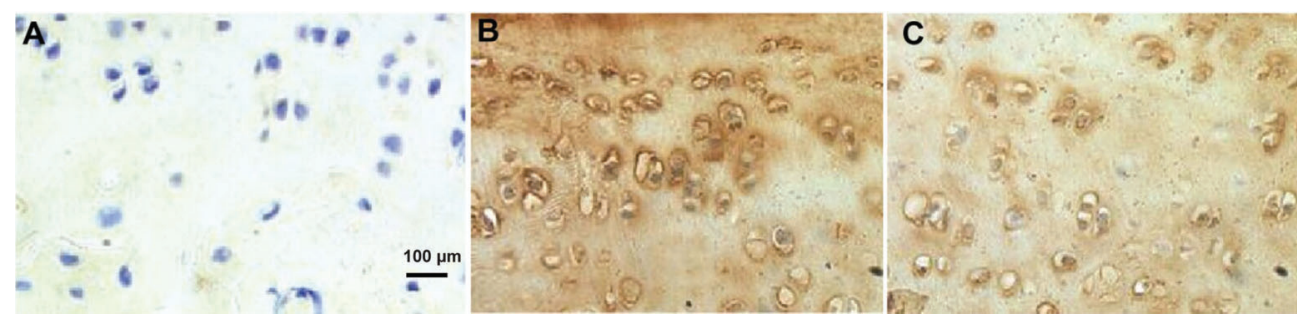

Figure 4. Immunohistochemical analysis of anti-tumor necrosis factor- $\alpha$ antibody (ATNF) effects on matrix metalloproteinase (MMP)-13 in cartilage lesions (original magnification $400 \times$ ). $A$, Staining for MMP-13 is less detectable in the sham-operated (SP) group. $B$, In the anterior cruciate ligament transection (ACLT) + normal saline (NS) group, MMP-13 expression is found throughout the articular cartilage. $C$, In the ACLT + ATNF group, MMP-13 is mainly detected in chondrocytes at and close to the articular surfaces.

ACLT injury compared with those in the ACLT+NS group.

Numerous studies support that the entire synovial joint is involved in OA, with alterations occurring in the articular cartilage, subchondral bone, capsule, ligaments, periarticular muscles, and synovial membrane $(26,27)$. However, articular cartilage is the major target of tissue injury with ulceration, fissures, and full-thickness loss from the joint surface (27). OA degeneration is also characterized by extensive joint remodeling, which is often associated with the formation of new bone (osteophytes) at the joint margins, increased subchondral plate thickness, and sclerosis (28). The rat ACLT model can only mimic some features of human $O A$, because human $O A$ is more complex and has different phases. However, articular cartilage degeneration is a hallmark of OA. Furthermore, recent studies have characterized the cartilage degradation in the rat ACLT model (29). In line with the previous studies, our study confirmed the effects of ATNF treatment on $\mathrm{OA}$, based on gross observations revealing that the majority of the cartilage surface had a smooth surface in the ACLT + ATNF group.

Knee instability following ACLT often induces OA accompanied by degradation of the articular cartilage matrix (29). Besides, Jean et al. (30) demonstrated that breakdown of the cartilage matrix can lead to fissures, fibrillation, gross ulceration, and even full-thickness loss at the joint surface. Aigner et al. (31) described that HE staining of OA cartilage shows surface fibrillation, typical chondrocyte clustering, and typical chondrocytes. Moreover, another study showed that TNF- $\alpha$ suppresses matrix synthesis by chondrocytes, which is essential for adequate matrix function and balance in RA and OA (32). In our study, we used HE staining and a modified Mankin score to evaluate the cartilage degeneration. Increases in the Mankin score indicated that degenerative changes had occurred (Figures 2 and 3). We also showed that both the macroscopic and Mankin scores were significantly lower in the ACLT+ATNF group than in the ACLT+NS group. Moreover, reduction in the severity of the structural changes and less Masson trichrome staining were observed in the ACLT + ATNF group. In addition, the present findings confirm and extend previous observations on the calcified zone of cartilage in experimental rat OA. Thus, we suggest that ATNF treatment may directly contribute to chondrocyte proliferation and ECM integrity.

Subchondral bone and articular cartilage act as a functional unit in joints (33). Subchondral bone has been shown to be an interesting target in OA treatment (34). Through microscopic observations, Radin et al. (35) showed that subchondral trabecular microdamage could aggravate cartilage degradation in OA. Muraoka et al. (36) reported that before the onset of cartilage degeneration in $\mathrm{OA}$, the subchondral bone was fragile, and had low BV/TV and Tb.Th, and high Tb.Sp. In our study, the histomorphometric analysis of subchondral bone showed that ATNF treatment markedly increased the BV/TV, Tb.Th, and Tb.N (Table 2), indicating that the subchondral bone microstructure was improved. Therefore, our results suggest that ATNF treatment might alter the subchondral bone quality by improving the subchondral trabecular microstructure in this rat model of OA.

MMPs are the major mediators of cartilage destruction and can break down the components of the ECM (37). Goldring et al. (38) demonstrated that MMP-13, which is the major type II collagen-degrading collagenase in cartilage ECM and can be induced by interleukin-1 $\beta$ and TNF- $\alpha$, contributed not only to irreversible joint damage in $\mathrm{OA}$, but also to the initiation/onset phase. In addition, another study revealed that TNF- $\alpha$ regulates MMP expression through signal transduction pathways, such as the nuclear factor- $\kappa B$ pathway (32). Besides, Kanbe et al. (39) demonstrated that adalimumab treatment for RA could decrease MMP-3 expression in the synovium. In the present study, immunohistochemical analyses demonstrated that ATNF treatment reduced the expression of MMP-13, and that both the numbers and integrated absorbance values of positive cells were lower in the ACLT + ATNF group than those in the $A C L T+N S$ group. Taken together, the present findings suggest a possible mechanism for adalimumab in 
counteracting $\mathrm{OA}$ that involves prevention of cartilage degeneration by inhibiting MMP-13 expression.

In summary, our findings strongly suggest that ATNF counteracts the histomorphological cartilage degeneration and subchondral bone loss associated with OA by decreasing the MMP-13 expression and improving the subchondral bone microstructure in a rat model of $O A$. The protective effects of ATNF treatment raise the possibility that this form of treatment may have therapeutic benefits for humans with knee $O A$, in agreement with the few previous studies in clinical settings. However, the present study cannot explain the concrete mechanism underlying the ATNF-induced

\section{References}

1. Peat G, McCarney R, Croft P. Knee pain and osteoarthritis in older adults: a review of community burden and current use of primary health care. Ann Rheum Dis 2001; 60: 91-97, doi: 10.1136/ard.60.2.91.

2. Abramson SB, Attur M. Developments in the scientific understanding of osteoarthritis. Arthritis Res Ther 2009; 11: 227, doi: 10.1186/ar2655.

3. Burr DB. Anatomy and physiology of the mineralized tissues: role in the pathogenesis of osteoarthrosis. Osteoarthritis Cartilage 2004; 12 (Suppl A): S20-S30, doi: 10.1016/ j.joca.2003.09.016.

4. Haseeb A, Haqqi TM. Immunopathogenesis of osteoarthritis. Clin Immunol 2013; 146: 185-196, doi: 10.1016/ j.clim.2012.12.011.

5. van den Berg WB. Osteoarthritis year 2010 in review: pathomechanisms. Osteoarthritis Cartilage 2011; 19: 338341, doi: 10.1016/j.joca.2011.01.022.

6. Cheng T, Zhang L, Fu X, Wang W, Xu H, Song H, et al. The potential protective effects of calcitonin involved in coordinating chondrocyte response, extracellular matrix, and subchondral trabecular bone in experimental osteoarthritis. Connect Tissue Res 2013; 54: 139-146, doi: 10.3109/ 03008207.2012.760549.

7. Brandt KD, Dieppe P, Radin E. Etiopathogenesis of osteoarthritis. Med Clin North Am 2009; 93: 1-24, xv, doi: 10.1016/j.mcna.2008.08.009.

8. Lane Smith $R$, Trindade $M C$, Ikenoue $T$, Mohtai $M$, Das P, Carter DR, et al. Effects of shear stress on articular chondrocyte metabolism. Biorheology 2000; 37: 95-107.

9. Kapoor M, Martel-Pelletier J, Lajeunesse D, Pelletier JP, Fahmi $H$. Role of proinflammatory cytokines in the pathophysiology of osteoarthritis. Nat Rev Rheumatol 2011; 7: 33-42, doi: 10.1038/nrrheum.2010.196.

10. Al-Rayes $\mathrm{H}, \mathrm{Al}$-Swailem R, Albelawi M, Arfin M, Al-Asmari A, Tariq M. TNF-alpha and TNF-beta Gene Polymorphism in Saudi Rheumatoid Arthritis Patients. Clin Med Insights Arthritis Musculoskelet Disord 2011; 4: 55-63.

11. Garces S, Demengeot J, Benito-Garcia E. The immunogenicity of anti-TNF therapy in immune-mediated inflammatory diseases: a systematic review of the literature with a meta-analysis. Ann Rheum Dis 2013; 72: 1947-1955, doi: 10.1136/annrheumdis-2012-202220. decrease in MMP-13 expression, and further efforts are needed to clarify this mechanism in future studies. Taken together, the present study suggests that adalimumab is a good candidate for limiting the pathological progress in OA.

\section{Acknowledgements}

This study was supported by National Natural Science Foundation of China (grant number: 30700853; 81371979) and Scientific Research Subject of Shanghai Manicipal Health and Family Planning Committee (grant number: 20144Y0258).
12. Herenius MM, Oliveira AS, Wijbrandts CA, Gerlag DM, Tak PP, Lebre MC. Anti-TNF therapy reduces serum levels of chemerin in rheumatoid arthritis: a new mechanism by which anti-TNF might reduce inflammation. PLoS One 2013; 8: e57802, doi: 10.1371/journal.pone.0057802.

13. Hoff M, Kvien TK, Kalvesten J, Elden A, Haugeberg G. Adalimumab therapy reduces hand bone loss in early rheumatoid arthritis: explorative analyses from the PREMIER study. Ann Rheum Dis 2009; 68: 1171-1176, doi: 10.1136/ard.2008.091264.

14. Moller Dohn U, Boonen A, Hetland ML, Hansen MS, Knudsen LS, Hansen A, et al. Erosive progression is minimal, but erosion healing rare, in patients with rheumatoid arthritis treated with adalimumab. A 1 year investigator-initiated follow-up study using high-resolution computed tomography as the primary outcome measure. Ann Rheum Dis 2009; 68: 1585-1590, doi: 10.1136/ ard.2008.097048.

15. Verbruggen G, Wittoek R, Vander CB, Elewaut D. Tumour necrosis factor blockade for the treatment of erosive osteoarthritis of the interphalangeal finger joints: a double blind, randomised trial on structure modification. Ann Rheum Dis 2012; 71: 891-898, doi: 10.1136/ard.2011.149849.

16. Stoop R, Buma $P$, van der Kraan PM, Hollander AP, Billinghurst RC, Meijers $\mathrm{TH}$, et al. Type II collagen degradation in articular cartilage fibrillation after anterior cruciate ligament transection in rats. Osteoarthritis Cartilage 2001; 9: 308-315, doi: 10.1053/joca.2000.0390.

17. Hayami T, Pickarski M, Wesolowski GA, McLane J, Bone A, Destefano J, et al. The role of subchondral bone remodeling in osteoarthritis: reduction of cartilage degeneration and prevention of osteophyte formation by alendronate in the rat anterior cruciate ligament transection model. Arthritis Rheum 2004; 50: 1193-1206, doi: 10.1002/(ISSN) $1529-0131$.

18. Lorenz J, Grässel S. Experimental osteoarthritis models in mice. Mouse genetics. city: Springer; 2014.

19. Stoop R, Buma P, van der Kraan PM, Hollander AP, Clark BR, Robin Poole A, et al. Differences in type II collagen degradation between peripheral and central cartilage of rat stifle joints after cranial cruciate ligament transection. Arthritis Rheum 2000; 43: 2121-2131, doi: 10.1002/15290131(200009)43:9 <2121::AID-ANR24 > 3.0.CO;2-N. 
20. von der Mark K, von der Mark $\mathrm{H}$. The role of three genetically distinct collagen types in endochondral ossification and calcification of cartilage. J Bone Joint Surg $\mathrm{Br} 1977$; 59-B: 458-464.

21. Mankin HJ, Dorfman H, Lippiello L, Zarins A. Biochemical and metabolic abnormalities in articular cartilage from osteoarthritic human hips. II. Correlation of morphology with biochemical and metabolic data. J Bone Joint Surg Am 1971; 53: 523-537.

22. Fitzpatrick LA, Turner RT, Ritman ER. Endochondral bone formation in the heart: a possible mechanism of coronary calcification. Endocrinology 2003; 144: 2214-2219, doi: 10.1210/en.2002-0170.

23. Ghasemi A, Zahediasl S. Normality tests for statistical analysis: a guide for non-statisticians. Int $J$ Endocrinol Metab 2012; 10: 486-489.

24. Struchalin MV, Dehghan A, Witteman JC, van Duijn C, Aulchenko YS. Variance heterogeneity analysis for detection of potentially interacting genetic loci: method and its limitations. BMC Genet 2010; 11: 92, doi: 10.1186/1471-2156-11-92.

25. Zhang $L$, Hu $H$, Tian $F$, Song $H$, Zhang $Y$. Enhancement of subchondral bone quality by alendronate administration for the reduction of cartilage degeneration in the early phase of experimental osteoarthritis. Clin Exp Med 2011; 11: 235243, doi: 10.1007/s10238-011-0131-z.

26. Malemud CJ. Cytokines as therapeutic targets for osteoarthritis. BioDrugs 2004; 18: 23-35, doi: 10.2165/00063030200418010-00003.

27. Nuki G. Role of mechanical factors in the aetiology, pathogenesis and progression of osteoarthritis. Osteoarthritis. Berlin: Springer; 1999.

28. Goldring MB, Goldring SR. Osteoarthritis. J Cell Physiol 2007; 213: 626-634.

29. Hayami T, Pickarski M, Zhuo Y, Wesolowski GA, Rodan GA, Duong LT. Characterization of articular cartilage and subchondral bone changes in the rat anterior cruciate ligament transection and meniscectomized models of osteoarthritis. Bone 2006; 38: 234-243, doi: 10.1016/j.bone.2005.08.007.

30. Jean $\mathrm{YH}$, Wen ZH, Chang YC, Hsieh SP, Tang CC, Wang $\mathrm{YH}$, et al. Intra-articular injection of the cyclooxygenase-2 inhibitor parecoxib attenuates osteoarthritis progression in anterior cruciate ligament-transected knee in rats: role of excitatory amino acids. Osteoarthritis Cartilage 2007; 15: 638-645, doi: 10.1016/j.joca.2006.11.008.

31. Aigner T, Hemmel M, Neureiter D, Gebhard PM, Zeiler G, Kirchner T, et al. Apoptotic cell death is not a widespread phenomenon in normal aging and osteoarthritis human articular knee cartilage: a study of proliferation, programmed cell death (apoptosis), and viability of chondrocytes in normal and osteoarthritic human knee cartilage. Arthritis Rheum 2001; 44: 1304-1312.

32. Seguin CA, Bernier SM. TNFalpha suppresses link protein and type II collagen expression in chondrocytes: Role of MEK1/2 and NF-kappaB signaling pathways. J Cell Physiol 2003; 197: 356-369.

33. Lories RJ, Luyten FP. The bone-cartilage unit in osteoarthritis. Nat Rev Rheumatol 2011; 7: 43-49, doi: 10.1038/ nrrheum.2010.197.

34. Neogi T. Clinical significance of bone changes in osteoarthritis. Ther Adv Musculoskelet Dis 2012; 4: 259-267, doi: 10.1177/1759720X12437354.

35. Radin EL, Rose RM. Role of subchondral bone in the initiation and progression of cartilage damage. Clin Orthop Relat Res 1986; 34-40.

36. Muraoka T, Hagino H, Okano T, Enokida M, Teshima R. Role of subchondral bone in osteoarthritis development: a comparative study of two strains of guinea pigs with and without spontaneously occurring osteoarthritis. Arthritis Rheum 2007; 56: 3366-3374.

37. Burrage PS, Mix KS, Brinckerhoff CE. Matrix metalloproteinases: role in arthritis. Front Biosci 2006; 11: 529-543, doi: 10.2741/1817

38. Goldring MB, Otero M, Plumb DA, Dragomir C, Favero M, El Hachem K, et al. Roles of inflammatory and anabolic cytokines in cartilage metabolism: signals and multiple effectors converge upon MMP-13 regulation in osteoarthritis. Eur Cell Mater 2011; 21: 202-220.

39. Kanbe K, Chiba J, Nakamura A. Decrease of CD68 and MMP-3 expression in synovium by treatment of adalimumab for rheumatoid arthritis. Int J Rheum Dis 2011; 14: 261-266. 